\title{
Pin Oak (Quercus palustris) Leaf Extract Mediated Synthesis of Triangular, Polyhedral and Spherical Gold Nanoparticles
}

\author{
Jonathan D. Judy ${ }^{1}$, N. V. K. V. Prasad Tollamadugu' ${ }^{2 *}$, Paul M. Bertsch ${ }^{1}$ \\ ${ }^{1}$ Department of Plant and Soil Sciences, University of Kentucky, Lexington, USA \\ ${ }^{2}$ Institute of Frontier Technology, Regional Agricultural Research Station, Acharya NG Ranga University, Tirupati, India \\ Email: ${ }^{t}$ tnvkvprasad@gmail.com
}

Received August 29, 2012; revised October 3, 2012; accepted October 15, 2012

\begin{abstract}
Fabrication of phytogenic metal nanoparticles is a developing area of nanobiotechnology which has economic and potentially eco-friendly advantages over conventional chemical and physical synthesis methods. We report the synthesis of anisotropic gold nanostructures using a phytoextract from the leaves of the pin oak tree, Quercus palustris. We used $2.5 \%, 5 \%$, and $10 \% \mathrm{~m} / \mathrm{v}$ extract concentrations to determine the importance of pin oak phytoextract concentration on the properties of the nanomaterials synthesized. Characterization of the resulting nanomaterials revealed that the concentration of the phytoextract is a key factor controlling the size and relative frequency of shapes the gold nanostructures formed. TEM micrographs demonstrate that triangular, spherical, and polyhedral gold nanostructures were formed in all treatments, although UV-Vis spectra indicated that the formation of fewer nanostructures in the $2.5 \% \mathrm{~m} / \mathrm{v}$ phytoextract treatment We found that anisotropic nanostructures are formed at the highest concentrations in the preparation using $10 \% \mathrm{~m} / \mathrm{v}$ phytoextract. UV-Vis, TEM, and DLS data indicate that the $5 \% \mathrm{~m} / \mathrm{v}$ phytoextract results in the synthesis of the smallest sized nanoparticles.
\end{abstract}

Keywords: Biosynthesis; Phytogenic; Phytonanotechnology; Biogenic; Anisotrops

\section{Introduction}

Nanoparticles (NPs) are of great interest due to their small size and large surface area to volume ratio. The most common method of synthesizing noble metal nanostructures is via reduction of the metal salt with a suitable reducing agent in the presence of a stabilizer [1]. However, the ability to synthesize anisotropic noble metal nanostructures with high yield remains a challenge as the prevailing protocol requires complex and time-consuming steps. Moreover, there is a growing demand for the development of clean, non-toxic, bio-compatible and ecofriendly methods for the synthesis of metal NPs. Biogenic synthesis methods are generally considered to be safe, cost-effective, and adaptable to the large scale production of metallic nanostructures.

Current trends in metal nanostructure synthesis focus on monodispersity of size and selectivity of shape, both of which are the key for manipulating the unique magnetic, catalytic, optoelectronic, and mechanical properties of nanomaterials [2]. Control of the particle shape and size is essential for modified surface plasmon resonance

${ }^{*}$ Corresponding author.
(SPR) properties exploited for the development of optical and near-infrared metamaterials [3]. Gold $(\mathrm{Au})$ and silver (Ag) nanomaterials exhibit important shape dependent properties $[2,4]$. Nanotriangles exhibit two or more prominent absorption bands, one a transverse absorption band in the visible region and the other a longitudinal absorption band in NIR region, which are similar to properties observed for nanorods [4,5]. The NIR region is of significant biological importance since it represents the only spectral window for both therapeutic and imaging applications in biological tissue which do not have significant absorption in the NIR region. Therefore, Au nanotriangles with strong NIR absorption could find potential applications in such areas as surface enhanced raman spectroscopy (SERS) substrates [6], infrared radiation absorbing optical coatings [7], and wave guides for electromagnetic radiation. Further, Au NPs have been used in protein assays [8], immunoassays [9], capillary electrophoresis [10], and as thermal scalpels to kill cancer cells $[11,12]$.

Biosynthesis of both isotropic and anisotropic NPs has been demonstrated with a variety of biomolecules including chitosan [13], enzymes [14] and plant extracts. Trian- 
gular and truncated triangular Au NPs were synthesized by Chandran et al. (2006) using Aloe vera plant extract [15]. Triangular Au NPs have also obtained by the reduction of Au ions by lemongrass extract [16]. In both studies, freshly cut plant material was boiled into a broth to synthesize the phytoextract, the concentration of which was found to influence NP size as well as the amount of triangular NPs produced. Compared to this broth, sundried/shade-dried biomass has the advantages of convenient preservation and storage in addition to requiring less energy to produce. Synthesis of anisotropic nanomaterials using sun or shade dried plant biomass has been reported by Ghosh et al. (2011) using Dioscorea bulbifera tuber extract [17] and by Fayez et al. (2011) using Cinnamomum camphora leaf extract [18]. In the former study, the researchers also found that the shape and size of the nanomaterials produced was influenced by reaction time and reaction temperature, with $50^{\circ} \mathrm{C}$ being the optimal temperature. The importance of reaction time and temperature in the formation of anisotropic nanostructures was also reported by Chili et al. (2008), who demonstrated polymer mediated synthesis of anisotropic $\mathrm{Au}$ NPs [19] and by Shao et al. (2004), who demonstrated aspartate mediated synthesis of triangular and hexagonal nanostructures under room temperature conditions [20] whereas the same reaction did not yield traingular and hexagonal structures when synthesized at a boil [21].

We have used a phytoextract from shade-dried leaves of the pin oak tree, Quercus palustris, at varying concentrations to synthesize $\mathrm{Au}$ nanostructures at room temperature. The objectives of the study were to investigate whether pin oak leaf extract from shade dried oak leaves will induce formation of anisotropic Au nanostructures at room temperature and to determine differences in particle size and shape as a function of phytoextract concentration. The results of this study are the first data evaluating the use of Quercus palustris extract for use in green nanomaterial synthesis and could have important implications for the development of cost-effective large scale production of anisotropic $\mathrm{Au}$ nanostructures.

\section{Materials and Methods}

Chloroauric acid $\left(\mathrm{HAuCl}_{4} \cdot 3 \mathrm{H}_{2} \mathrm{O}\right)$ was purchased from Sigma-Aldrich and used as received. Pin oak leaves (Quercus palustris) were collected in August from the Lexington Arboretum in Lexington, Kentucky USA. Pin oak is a common ornamental tree native to the eastern United States with limited economic importance [22]. Leaves were thoroughly washed with tap water and allowed to air dry in the shade, after which the leaf tissue was removed from the stems. The leaf tissue was finely ground using a mechanical plant tissue grinder. Oak leaf extract was prepared by adding $2.5,5$, or $10 \mathrm{~g}$ ground oak leaves to $100 \mathrm{~mL}$ doubly deionized water (DDI) water and heated to $100^{\circ} \mathrm{C}$ for 45 minutes. Following the extraction solutions were allowed to cool to room temperature.

Five $\mathrm{mL}$ of $2.5 \%, 5.0 \%$, and $10.0 \% \mathrm{~m} / \mathrm{v}$ oak leaf extract were added to $95 \mathrm{~mL}$ of $10^{-3} \mathrm{M}$ aqueous $\mathrm{HAuCl}_{4} \cdot 3 \mathrm{H}_{2} \mathrm{O}$ solution. The reaction mixtures were allowed to stand at room temperature for $24 \mathrm{~h}$. Suspensions were centrifuged at $2250 \times \mathrm{g}$ for $10 \mathrm{~min}$ and the supernatant decanted. The concentration of the resulting suspension was determined by dissolving an aliquot of each suspension in concentrated aqua regia and measuring the amount of Au present in a diluted sample via inductively coupled plasma mass spectrometry (ICP-MS) using an Agilent 7500 cx ICP-MS (Agilent, Santa-Clara, CA, USA). Additionally, $3 \mathrm{~mL}$ aliquots of each nanoparticle suspension were filtered through a $3 \mathrm{kDa}$ Amicon Ultra membrane (Millipore, Billerica, MA). These samples were also analyzed for Au using ICP-MS to determine the concentration of dissolved $\mathrm{Au}$ in the nanomaterial suspensions.

UV-Vis spectroscopic measurements of the synthesized $\mathrm{Au}$ nanostructures were carried out on a Varian model 50 Bio dual-beam spectrophotometer (Agilent, Santa-Clara, CA, USA) operated at a resolution of $1 \mathrm{~nm}$. Hydrodynamic diameter and electrophoretic mobility measurements of the Au nanostructures were measured with a Nano-ZS zetasizer using $173^{\circ}$ backscatter analysis method (Malvern, Worcestershire, UK). FT-IR measurements of the oak leaf extract and Au NPs synthesized using different concentrations of the extract were carried out on a Thermo-Nicolet 6700 (Thermo Scientific, Waltham, MA, USA) in the diffuse reflectance mode operated at a resolution of $8 \mathrm{~cm}^{-1}$. Filtrate samples were also analyzed with filtrate spectra subtracted from suspension spectra. TEM samples were prepared by pipetting drops of each NP suspension on 200 mesh carbon coated formvar copper grids and allowing the sample to dry by evaporation. TEM size analysis of the NPs was performed using a Jeol 2010F HR-TEM operating at 200 $\mathrm{keV}$. Particle size and shape distributions were quantified based on measurements of at least 100 particles from at least 3 different micrographs using ImageJ software.

\section{Results and Discussion}

After adding the phytoextract to the Au chloride solutions, the color of the solution changed from pale yellow to dark red immediately on mixing in all treatments, indicating formation of Au nanostructures. The SPR band observed near $530 \mathrm{~nm} \mathrm{[11]} \mathrm{in} \mathrm{all} \mathrm{three} \mathrm{treatments} \mathrm{con-}$ firms the reduction of $\mathrm{Au}^{3+}$ and the formation of Au NPs (Figure 1). However, despite the fact that a peak exists 


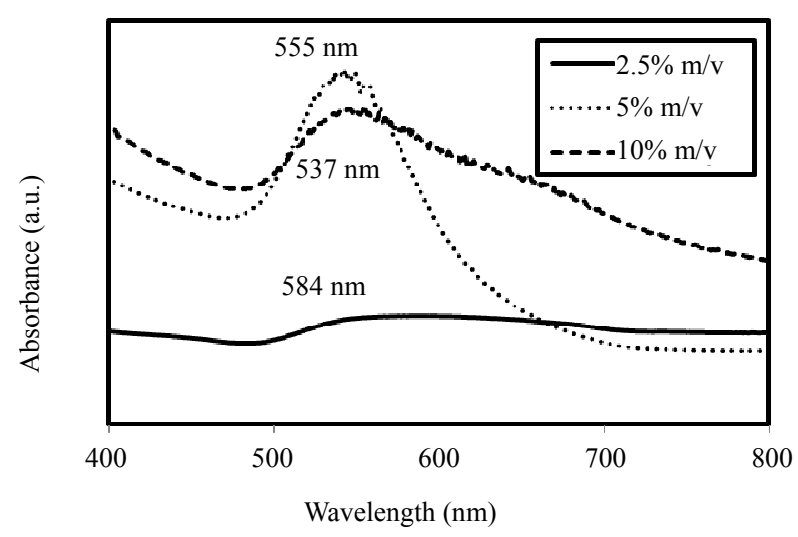

Figure 1. UV-Vis spectra for nanoparticle suspensions synthesized using 2.5 (solid line), 5 (dotted line), and $10 \%$ (dashed line) $\mathrm{m} / \mathrm{v}$ plant extract. Peak absorbance for each spectrum is labeled.

in all treatments near $530 \mathrm{~nm}$, the peak for the $2.5 \% \mathrm{~m} / \mathrm{v}$ phytoextract treatment is very weak, suggesting that fewer nanostructures were formed in this treatment that in the $5 \%$ and $10 \% \mathrm{~m} / \mathrm{v}$ treatments. The peak absorbance for the nanoparticle suspensions occurs at 584, 537, and $555 \mathrm{~nm}$ for the $2.5 \%, 5 \%$, and $10 \% \mathrm{~m} / \mathrm{v}$ treatments, respect-tively. The frequency and width of the SPR absorption peak depends on the size and shape of the metal NPs as well as on the dielectric constant on the metal itself and the surrounding medium [23]. For Au NPs with a mean diameter larger than $25 \mathrm{~nm}$, the absorbance maximum of the SPR band increases with increasing mean NP diameter $[23,24]$. For Au NPs with a mean diameter smaller than $25 \mathrm{~nm}$, the trend reverses and the SPR band absorbance maximum increases with decreasing mean NP diameter [24]. Alternately, the increase in absorbance wavelength for the $2.5 \% \mathrm{~m} / \mathrm{v}$ treatment could be indicative of a mean diameter smaller than $25 \mathrm{~nm}$. Since the peak absorbance for the nanomaterial suspendsion synthesized with $5 \% \mathrm{~m} / \mathrm{v}$ smallest phytoextract occurred at the shortest wavelength, these data indicate that the $5 \% \mathrm{~m} / \mathrm{v}$ phytoextract yielded the NPs. The absorption spectrum for the $10 \% \mathrm{~m} / \mathrm{v}$ treatment is broad approaching the NIR region compared to the other two treatments, suggesting a relatively high amount of anisotropic NPs [7]. This result differs from other studies examining the effect of increased phytoextract concentration on nanomaterial synthesis that found that particles synthesized with high concentrations of phytoextract had minimal absorbance in the NIR region [7,15]. Although the reasons for these contradictions are unclear, they are possibly related to the relationship between NP suspension surface area and phytoextract concentration used in each individual study. We speculate that increasing phytoextract concentration will stabilize NP suspensions to a point corresponding to adequate NP surface coverage, after which increasing phytoextract concentration will begin to destabilize the suspension and promote aggregation, increasing NP size and resulting in a red shift in UV-Vis absorbance. This hypothesis is supported by a recent study that reported a decrease in NP size and a blue shift in absorbance as a result of increasing the volume of olive leaf phytoextract added from 0.2 to $2 \mathrm{~mL}$ but an increase in particle size and red shift in absorbance when the volume of phytoextract added was increased from 3 to $6 \mathrm{~mL}$ [25].

Measurements of electrophoretic mobility reveal that the nanoparticle suspensions synthesized with 2.5 and $5 \% \mathrm{~m} / \mathrm{v}$ phytoextract have relatively similar zeta potentials, -34.2 and $-41.9 \mathrm{mV}$, respectively. Hydrodynamic diameter measurements reveal mean diameters of 124.4, 46.1 , and $50.2 \mathrm{~nm}$ for the $2.5,5$, and $10 \% \mathrm{~m} / \mathrm{v}$ treatments, respectively. The polydispersity index of the NPs increases with increasing phytoextract concentration (Table 1). The negative zeta potential values revealed by electrophoretic mobility measurements are indicative of high negative surface charges which provide stabilization of the particles in the form of electrostatic repulsion from other particles. However, the suspension synthesized with $10 \% \mathrm{~m} / \mathrm{v}$ phytoextract has a zeta potential of only $-13.8 \mathrm{mV}$, indicating these particles have a lower degree of charge stabilization.

FTIR spectra reveal peaks at approximately 780,1635 , 3250 , and $3460 \mathrm{~cm}^{-1}$ in the phytoextract as well as in all three treatments (Figure 2(a)). FTIR spectra from suspension filtrate were subtracted from these initial spectra to investigate the molecules specifically associated with the nanomaterials (Figure 2(b)). In these subtraction spectra, only peaks at approximately 780 and $3250 \mathrm{~cm}^{-1}$ are present. In FTIR spectra of the original samples, the sharp peaks at approximately $3460 \mathrm{~cm}^{-1}$ and the peak at approximately $1635 \mathrm{~cm}^{-1}$ are both likely due to the presence of N-H bonds in either amide or amine groups [25]. In the subtraction spectra, the peak at $3250 \mathrm{~cm}^{-1}$ is likely due to the presence of phenolic hydroxyl groups [15] and the peak near $780 \mathrm{~cm}^{-1}$ likely due to the presence of aromatic compounds [26]. This result indicates that molecules with these functional groups are associated with the NPs.

TEM images of the NPs synthesized with the $2.5 \%$ $\mathrm{m} / \mathrm{v}$ phytoextract reveal that the suspension was predominately composed of masses of heavily aggregated nanostructures (Figures 3(a)-(c)). These structures were not used in the calculation of mean diameters and the distribution of shapes within the suspension. Superimposed on these is a relatively sparse population of larger nanospheres and anisotropic nanostructures with a mean diameter and standard deviation of $58.2 \pm 20.9 \mathrm{~nm}$ (Table 2). The nanoparticle suspension synthesized with the $5 \% \mathrm{~m} / \mathrm{v}$ phytoextract consists predominantly of spheres with 

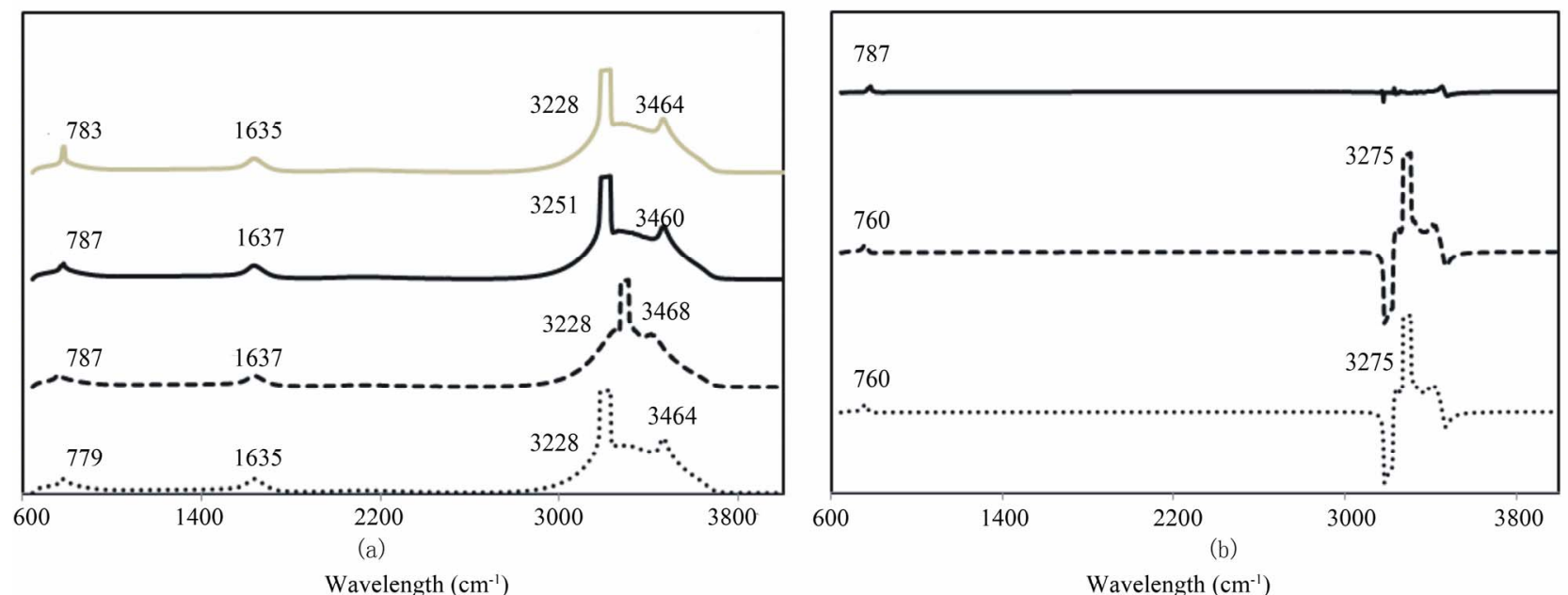

Wavelength $\left(\mathrm{cm}^{-1}\right)$

Wavelength $\left(\mathrm{cm}^{-1}\right)$

Figure 2. (a) FTIR spectra characterizing nanoparticle suspensions and oak leaf extract (b) Suspension spectra with filtrate spectra subtracted.

Table 1. Nanomaterial characterization data. Z-average diameter is equal to the hydrodynamic diameter based on light scattering intensity. PDI = polydispersivity index.

\begin{tabular}{|c|c|c|c|c|c|}
\hline $\begin{array}{l}\text { Plant Extract Concentration } \\
\qquad(\mathrm{m} / \mathrm{v})\end{array}$ & $\begin{array}{l}\text { Z-Average diameter } \\
(\mathrm{nm})\end{array}$ & $\begin{array}{l}\text { Zeta potential } \\
\quad(\mathrm{mV})\end{array}$ & $\mathrm{pH}$ & PDI & $\begin{array}{l}\text { Dissolved Au } \\
(\%, \mathrm{wt} / \mathrm{wt})\end{array}$ \\
\hline 2.5 & 124.4 & -41.9 & 2.4 & 0.16 & $6.8 \times 10^{-8}$ \\
\hline 5 & 46.1 & -34.2 & 2.5 & 0.31 & $3.6 \times 10^{-8}$ \\
\hline 10 & 50.2 & -13.8 & 2.7 & 0.48 & $4.2 \times 10^{-8}$ \\
\hline
\end{tabular}

Table 2. Summary of TEM data characterizing the size and shape distribution of the nanoparticle suspensions.

\begin{tabular}{cccc}
\hline $\begin{array}{c}\text { Plant Extract Concentration } \\
(\mathrm{m} / \mathrm{v})\end{array}$ & $\begin{array}{c}\text { Spheres/polyhedrons } \\
\text { (mean diameter }(\mathrm{nm}) \pm \mathrm{SD} \text {, relative } \\
\text { abundance) }\end{array}$ & $\begin{array}{c}\text { Triangles } \\
\text { (mean height }(\mathrm{nm}) \pm \mathrm{SD}, \\
\text { relative abundance) }\end{array}$ & $\begin{array}{c}\text { Other shapes, } \\
\text { (relative abundance) }\end{array}$ \\
\hline 2.5 & $58.2 \pm 20.9,89.8 \%$ & $216 \pm 62.8,5.1 \%$ & $\begin{array}{c}\text { Trapezoids, diamonds, } \\
5.1 \%\end{array}$ \\
5 & $6.4 \pm 1.6,>99 \% ; 35.8 \pm 15.6,<1 \%$ & $84.7 \pm 39.7,<1 \%$ & None observed \\
10 & $22.6 \pm 19.2,91.7 \%$ & $59.0 \pm 62.5,7.8 \%$ & Hexagons, $0.5 \%$ \\
\hline
\end{tabular}

a mean diameter and standard deviation of $6.4 \mathrm{~nm} \pm 1.6$. However, a second population of NPs consisting of larger spheres and triangles is also present, albeit in small numbers (Figures 3(d)-(f), Table 2). Overall, the particles in this suspension are smaller than nanostructures in the $2.5 \%$ and $10 \% \mathrm{~m} / \mathrm{v}$ treatments, consistent with the UV-Vis and DLS data. The nanoparticle suspension synthesized with the $10 \% \mathrm{~m} / \mathrm{v}$ phytoextract is mainly composed of a spherical/polyhedral population with a mean diameter and standard deviation of $22.6 \pm 19.2 \mathrm{~nm}$. However, a significant quantity of anisotropic nanostructures formed in this suspension with approximately $8 \%$ of the particles present as either triangular, hexagonal, or truncated triangles (Figures 3(g)-(i), Table 1). Unlike the NPs synthesized with the 2.5 and $5 \% \mathrm{~m} / \mathrm{v}$ phytoextract, there is no evidence for two different size populations of NPs in the $10 \% \mathrm{~m} / \mathrm{v}$ suspension.

TEM micrographs revealed the synthesis of anistotropic nanomaterials in each treatment, although there were large differences between NPs depending on treatment. The $5 \% \mathrm{~m} / \mathrm{v}$ extract produced the smallest NPs and the $10 \% \mathrm{~m} / \mathrm{v}$ extract produced the highest proportion of anisotropic nanostructures. Wiley and co-workers (2005) observed that nanorods evolve from multiply twinned 


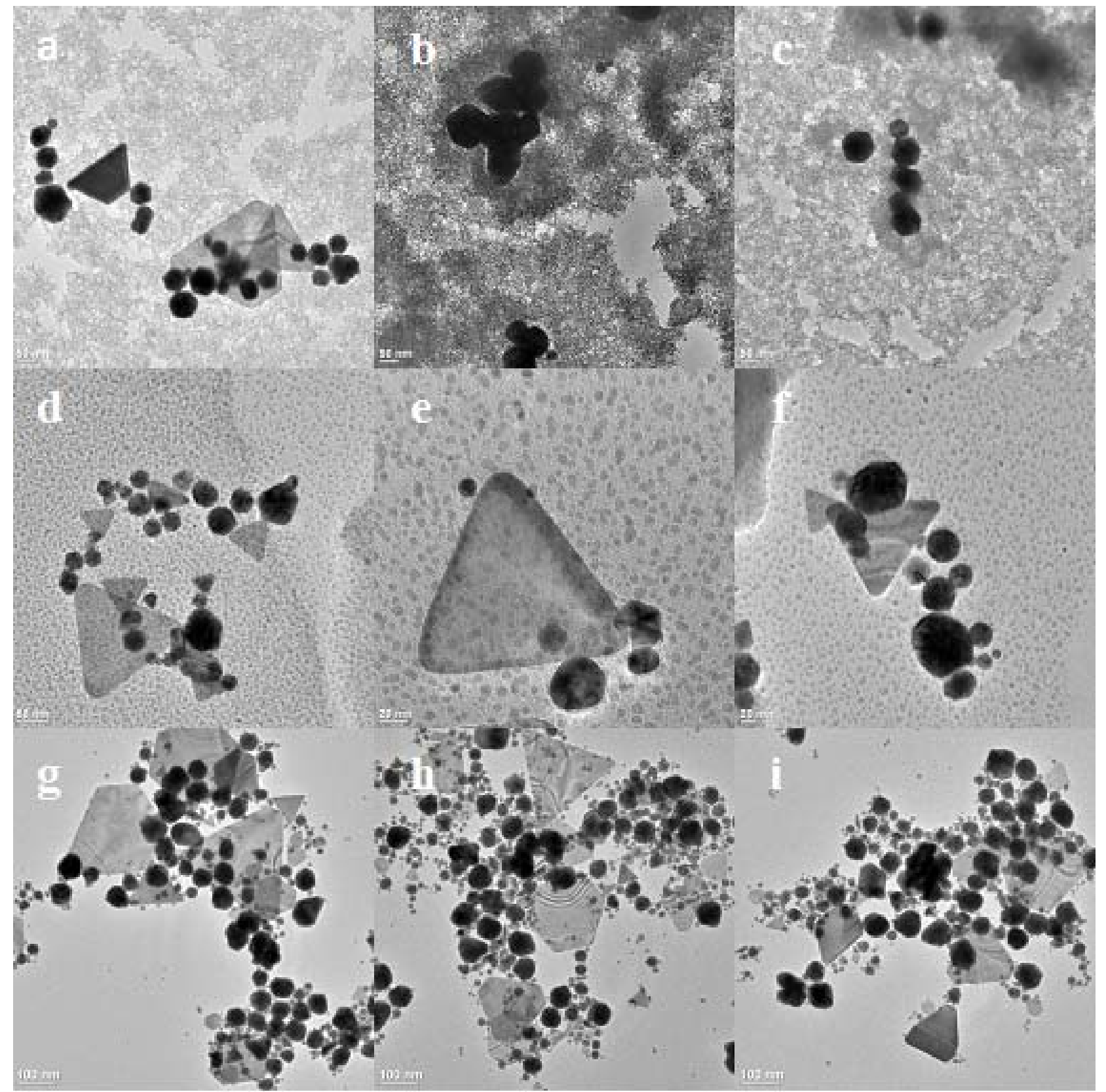

Figure 3. TEM micrographs of gold nanoparticles synthesized using (a-c) 2.5 (d-f) 5 and (g-i) $10 \%$ m/v phytoextract.

particles (MTPs) as a result of anisotropic growth caused by certain shape-directing agents [27]. We speculate that, in our study, the anisotropic structures observed could result from MTPs initially formed under slow reaction conditions that subsequently undergo shape transformations, eventually evolving into $\mathrm{Au}$ anisotropic structures as a result of the shape-directing constituents within the oak leaf extract. We speculate that a higher concentration of these shape directing agents in the $10 \% \mathrm{~m} / \mathrm{v}$ extract treatment resulted in the higher amount of anisotropic nanostructures. The sintering of spherical NPs at room temperature may also lead to the formation of anisotropic structures. A similar kind of crystal growth was observed under time dependent electron beam exposure to the $\mathrm{Au}$ NPs made with 2.5\% extract (Figure 4). It is possible that spherical Au NPs were initially formed at the reaction products which evolved to $\mathrm{Au}$ nanotriangles and polyhedral nanostructures as a result of reduction, assembly, and room temperature sintering [28]. Alternatively, the difference in the growth rates of the various crystallographic planes could direct the morphology of the NPs [29]. The formation of different structures likely results from triangular platelets forming when there is one twinning plane parallel to the plane of the platelet 


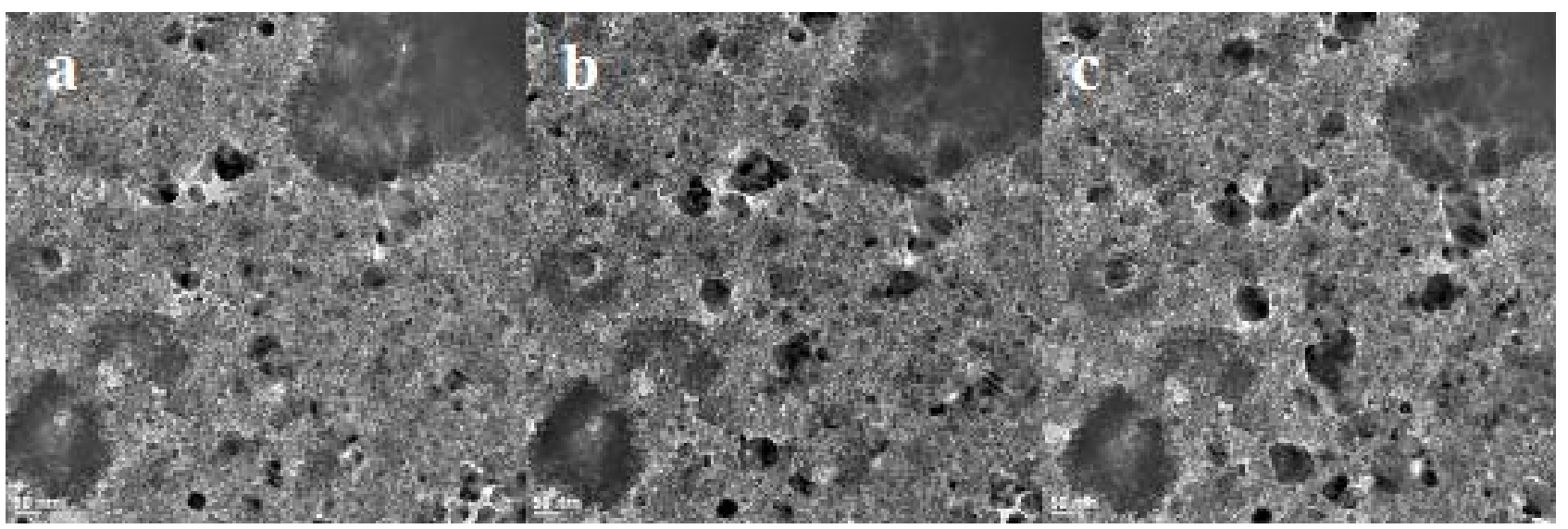

Figure 4. TEM micrographs of the response of gold nanoparticles synthesized using $2.5 \% \mathrm{~m} / \mathrm{v}$ oak extract to exposure to the electron beam; (a) $t=0$, (b) $t=60 \mathrm{~s}$, (c) $t=180 \mathrm{~s}$.

resulting in one set of fast growing concave edges [3], similar to the silver halide model [30,31]. Hexagonal platelets have been shown to result from two twinning planes which yield more highly symmetric growth $[30,31]$.

\section{Conclusions}

We have demonstrated for the first time the room temperature synthesis of $\mathrm{Au}$ nanospheres, nanopolyhedrons, and nanotriangles using phytoextract from shade-dried leaves from Quercus palustris. This synthesis protocol allows for the substitution of oak leaf phytoextract, a relatively eco-friendly and inexpensive bioreductant, for chemical reductants such as sodium citrate or sodium borohydride. Additionally, we have demonstrated the ability to synthesize Au NPs using shade dried leaves from a plant with little current economic importance. We have also demonstrated that manipulation of nanoparticle size and shape can be achieved by adjusting the concentration of oak phytoextract. The results of this study could have important practical implications for the development of cost-effective and green processes for the large scale production of anisotropic Au nanostructures.

\section{Acknowledgements}

Major funding for this research was provided by the National Science Foundation (NSF) and the Environmental Protection Agency (EPA) under NSF Cooperative Agreement EF-0830093, Center for the Environmental Implications of Nanotechnology (CEINT). This research was also partially supported by a grant from the U.S. Environmental Protection Agency's Sci- ence to Achieve Results (STAR) program (RD834574), the Transatlantic Initiative for Nanotech- nology and the Environment (TĪNE). Any opinions, findings, conclu- sions or recommendations expressed in this material are those of the author(s) and do not necessarily reflect the views of the NSF or the EPA. This work has not been subjected to NSF or EPA review and no official endorsement should be inferred. Additional funding was provided by Indian Council of Agricultural Research, New Delhi, India, under National Agricultural Innova- tion Project. The information reported in this paper (No. 12-06-110) is a product of the Kentucky Agricultural Experiment Station and is published with the approval of the Director.

\section{REFERENCES}

[1] J. Turkevich, P. C. Stevenson and J. A. Hillier, "A Study of the Nucleation and Growth Processes in the Synthesis of Colloidal Gold," Discussions of the Faraday Society, Vol. 11, 1951, pp. 55-75.

[2] M. A. El-Sayed, "Some Interesting Properties of Metals Confined in Time and Nanometer Space of Different Shapes," Accounts of Chemical Research, Vol. 34, No. 4, 2001, pp. 257- 264.

[3] J. S. Evans, N. C. Beier and I. I. Smalyukh, "Alignment of High-Aspect Ratio Colloidal Gold Nanoplatelets in Nematic Liquid Crystals," Journal of Applied Physics, Vol. 110, No. 3, 2011, p. 033535.

[4] K. L. Kelly, E. Coronado, L. L. Zhao and G. C. Schatz, "The Optical Properties of Metal Nanoparticles: The Influence of Size, Shape, and Dielectric Environment," The Journal of Physical Chemistry B," Vol. 107, No. 3, 2003, pp. 668-677.

[5] L. J. Sherry, R. Jin, C. A. Mirkin, G. C. Schatz and R. P. Van Duyne, "Localized Surface Plasmon Resonance Spectroscopy of Single Silver Triangular Nanoprisms," Nano Letters, Vol. 6 No. 9, 2006, pp. 2060-2065. doi:10.1021/n1061286u

[6] L. A. Dick, A. D. McFarland, C. L. Haynes and R. P. Van Duyne, "Metal Film over Nanospheres (MFON) Electrodes for Surface-Enhanced Raman Spectroscopy (SERS): Improvements in Surface Nanostructure Stability and Suppression of Irreversible Loss," The Journal of Phy- 
sical Chemistry B, Vol. 106, No. 4, 2002, pp. 853- 860.

[7] S. S. Shankar, A. Rai, A. Ahmad and M. Sastry, "Controlling the Optical Properties of Lemongrass Ex- tract Synthesized Gold Nanotriangles and Potential Application in Infrared-Absorbing Optical Coatings," Chemistry of Materials, Vol. 17, No. 3, 2005, pp. 566- 572.

[8] D. Thang, R. Yuan and Y. Chai, "Biochemical and Immunochemical Characterization of the Antigen-Antibody Reaction on a Non-Toxic Biomimetic Interface Immobilized Red Blood Cells of Crucian Carp and Gold Nanoparticles," Biosensors and Bioelectronics, Vol. 22, No. 6, 2007, pp. 1116-1120.

[9] X. Liu, Q. Dai, L. Austin, J. Coutts, G. Knowles, J. Zou, H. Chen and Q. Huo, "Nanodlsa: A Novel Homogeneous Immunoassay for Biomarker Detection Using Gold Nanoparticles Coupled with Dynamic Light Scattering Detection," Journal of the American Chemical Society, Vol. 130, No. 9, 2008, pp. 2780-2782.

[10] W. L. Tseng, M. F. Huang, Y. F. Huang and H. T. Chang, "Nanoparticle-Filled Capillary Electrophoresis for the Separation of Long DNA Molecules in the Presence of Hydrodynamic and Electrokinetic Forces," Electrophoresis, Vol. 26, No. 16, 2005, pp. 3069- 3075.

[11] I. H. El-Sayed, X. Huang and M. A. El-Sayed, "Selective Laser Photo-Thermal Therapy of Epithelial Carcinoma Using Anti-EGFR Antibody Conjugated Gold Nanoparticles," Cancer Letters, Vol. 239, No. 1, 2006, pp. 129-135.

[12] O. Salata, "Applications of Nanoparticles in Biology and Medicine," Journal of Nanobiotechnology, Vol. 2 No. 1. 2004, pp. 3-6

[13] K. Yang, X. Wang, Z. Zhou, J. Xu, J. Weng and Q. Zhang, "One-Step Synthesis and Characterization of Chitosan-Mediated Micro-Sized Gold Nanoplates through a Thermal Process," IET Nanobiotechnology, Vol. 1, No. 6, 2007, pp. 107-111.

[14] I. Willner, R. Baron and B. Willner, "Growing Metal Nanoparticles by Enzymes," Advanced Materials Letters, Vol. 18, No. 9, 2006, pp. 1109-1120.

[15] S. P. Chandran, M. Chaudhary, R. Pasricha, A. Ahmad and M. Sastry, "Synthesis of Gold Nanotriangles and Silver Nanoparticles Using Aloe Vera Plant Extract," Biotechnology Progress, Vol. 22, No. 2, 2006, pp. 577-583. doi:10.1021/bp0501423

[16] A. Rai, M. Chaudhary, A. Ahmad, S. Bhargava and M. Sastry, "Synthesis of Triangular Au Core-Ag Shell Nanoparticles," Materials Research Bulletin, Vol. 42, No. 7, 2007, pp. 1212-1220.

[17] S. Ghosh, S. Patil, M. Ahire, R. Kitture, A. Jabgunde, S. Kale, K. Pardesi, J. Bellare, D. D. Dhavale and A. B. Chopade, "Synthesis of Gold Nanoanisotrops Using Dioscorea Bulbifera Tuber Extract," Journal of Nanoma- terials, Vol. 2011, No. 45, 2011, pp. 1-8.

[18] J. Huang, Q. Li, D. Sun, Y. Lu, Y. Su, X. Yang, H. Wang, Y. Wang, W. Shao, N. He, J. Hong and C. Chen, "Bio- synthesis of Silver and Gold Nanoparticles by Novel Sundried Cinnamomum Camphora Leaf," Nanotechnology, Vol. 18, No. 10, 2007, pp.

[19] M. M. Chili and N. Revaprasadu, "Synthesis of Anisotropic Gold Nanoparticles in a Water-Soluble Poly- mer," Materials Letters, Vol. 62, No. 23, 2008, pp. 3896- 3899.

[20] Y. Shao, Y. Jin and S. Dong, "Synthesis of Gold Nanoplates by Aspartate Reduction of Gold Chloride," Chemical Communications, Vol. 10, No. 9, 2004, pp. 1104-1105.

[21] S. Mandal, P. R. Selvakannan, S. Phadtare, R. Pasricha and M. Sastry, "Synthesis of a Stable Gold Hydrosol by the Reduction of Chloroaurate Ions by the Amino Acid, Aspartic Acid," Proceedings of the Indian Academy of Sciences, Chemical Sciences, Vol. 114, No. 4, 2002, pp. 513-520.

[22] J. H. Carey, “Quercus palustris,” 2012. http://www.fs.fed.us/database/feis/plants/tree/quepal/all.ht $\mathrm{ml}$.

[23] W. Cai, J. Hofmeister and T. Rainder, "Surface Effect on the Size Evolution of Surface Plasmon Resonances of Ag and $\mathrm{Au}$ Nanoparticles Dispersed within Mesoporous Silica," Physica E, Vol. 11, No. 4, 2001, pp. 339-344.

[24] S. Link and M. A. El-Sayed, "Size and Temperature Dependence of the Plasmon Absorption of Colloidal Gold Nanoparticles," The Journal of Physical Chemistry B, Vol. 103, No. 21, 1999, pp. 4212-4217.

[25] M. M. H. Khalil, E. H. Ismail and F. El-Magdoub, "Biosynthesis of $\mathrm{Au}$ Nanoparticles Using Olive Leaf Extract: 1st Nano Updates," Arabian Journal of Chemis- try, Vol. 5 No. 4, 2012, pp. 431-437. doi:10.1016/j.arabjc.2010.11.011

[26] D. A. Skoog, F. J. Holler and S. R. Crouch, "Instrumental Analysis," Cengage Learning, New Delhi, 2007.

[27] B. Wiley, Y. Sun, B. Mayers and Y. Xia, "Shape Controlled Synthesis of Metal Nanostructures: Yhe Case of Silver," European Journal of Medicinal Chemistry, Vol. 11, No. 2, 2005, pp. 454-463.

[28] S. S. Shankar, A. Rai, B. Ankamwar, A. Singh, A. Ahmad and M. Sastry, "Biological Synthesis of Triangular Gold Nanoprisms," Nature Materials, 2004, Vol. 3, No. 7, pp. 482-488.

[29] Z. L. Wang, "Transmission Electron Microscopy of Shape-Controlled Nanocrystals and Their Assemblies," The Journal of Physical Chemistry B, Vol. 104, No. 6, 2000, pp. 1153-1175.

[30] M. Grzelezak, J. Juste, P. Mulvaney and L. Liz-Marzan, "Shape Control in Gold Nanoparticle Synthesis," Chemical Society Reviews, Vol. 37, No. 9, 2008, pp. 1783-1791.

[31] C. Lofton and W. Sigmund "Mechanisms Controlling Crystal Habits of Gold and Silver Colloids," Advanced Functional Materials, Vol. 15 No. 7, 2005, pp. 1197-1208. doi:10.1002/adfm.200400091 